\title{
Frizzled-8 receptor is activated by the Wnt-2 ligand in non-small cell lung cancer
}

\author{
Dawn T Bravo, Yi-Lin Yang, Kristopher Kuchenbecker, Ming-Szu Hung, Zhidong Xu, David M Jablons \\ and Liang You*
}

\begin{abstract}
Background: Wnt-2 plays an oncogenic role in cancer, but which Frizzled receptor(s) mediates the Wnt-2 signaling pathway in lung cancer remains unclear. We sought to (1) identify and evaluate the activation of Wnt-2 signaling through Frizzled-8 in non-small cell lung cancer, and (2) test whether a novel expression construct dominant negative Wnt-2 (dnhWnt-2) reduces tumor growth in a colony formation assay and in a xenograft mouse model.

Methods: Semi-quantitative RT-PCR was used to identify the expression of Wnt-2 and Frizzled-8 in 50 lung cancer tissues from patients. The TCF reporter assay (TOP/FOP) was used to detect the activation of the Wnt canonical pathway in vitro. A novel dnhWnt-2 construct was designed and used to inhibit activation of Wnt-2 signaling through Frizzled-8 in 293T, 293, A549 and A427 cells and in a xenograft mouse model. Statistical comparisons were made using Student's t-test.

Results: Among the 50 lung cancer samples, we identified a 91\% correlation between the transcriptional increase of Wnt-2 and Frizzled-8 $(p<0.05)$. The Wnt canonical pathway was activated when both Wnt-2 and Frizzled-8 were co-expressed in 293T, 293, A549 and A427 cells. The dnhWnt-2 construct we used inhibited the activation of Wnt-2 signaling in 293T, 293, A549 and A427 cells, and reduced the colony formation of NSCLC cells when $\beta$-catenin was present $(p<0.05)$. Inhibition of Wnt-2 activation by the dnhWnt-2 construct further reduced the size and mass of tumors in the xenograft mouse model $(p<0.05)$. The inhibition also decreased the expression of target genes of Wnt signaling in these tumors.

Conclusions: We demonstrated an activation of Wnt-2 signaling via the Frizzled-8 receptor in NSCLC cells. A novel dnhWnt-2 construct significantly inhibits Wnt-2 signaling, reduces colony formation of NSCLC cells in vitro and tumor growth in a xenograft mouse model. The dnhWnt-2 construct may provide a new therapeutic avenue for targeting the Wnt pathway in lung cancer.
\end{abstract}

Keywords: Frizzled-8, Wnt-2, dnhWnt-2 Construct, Lung Cancer, Wnt Signaling

\section{Background}

Lung cancer is the most commonly diagnosed malignancy worldwide and is responsible for over one million deaths each year $[1,2]$. Current treatment strategies include surgical resection, chemotherapy, radiation therapy, targeted therapy, or a combination of treatments, depending on disease type and stage [1,2]. Despite advances in multimodality treatments, lung cancer remains

\footnotetext{
* Correspondence: liang.you@ucsfmedctr.org

Department of Surgery, Helen Diller Family Comprehensive Cancer Center, University of California, 2340 Sutter Street, N-221, San Francisco, CA 94115,
} USA

\section{Biomed Central}

highly lethal, with a 5 -year survival rate of less than $15 \%$ [2]. New treatment strategies are urgently needed.

Wnt signaling elicits numerous cellular responses including self-renewals of stem cells [3]. Currently, 10 Frizzled proteins have been identified in mammals as the receptors for Wnt proteins. Transduction of Wnt signaling begins when Wnt ligands bind to the cysteinerich Wnt binding domain (CRD) of Frizzled receptors at the cell membrane and initiate either the 'canonical' or 'non-canonical' pathways [4]. The canonical Wnt signaling pathway regulates the stability of $\beta$-catenin [5]. When Wnt is not activated, $\beta$-catenin is phosphorylated by the destruction complex and degraded 
by ubiquitination [6,7]. When binding to Frizzled receptors and low-density lipoprotein co-receptors 5 and 6 (LRP5/6) on cell membrane [8,9], Wnt signaling is activated and Dishevelled (DVL) recruits the destruction complex to the plasma membrane [10], resulting in $\beta$-catenin stabilization and subsequent accumulation in the cytoplasm [5]. Stabilized $\beta$-catenin then enters the cell nucleus and associates with lymphoid enhancer-binding factor (LEF)/T-cell factor (TCF) transcription factors $[11,12]$ to promote transcription of important downstream target genes, many of which have been implicated in cancer [13-15]. Aberrant activation caused by $\beta$-catenin or APC mutations leads to the constitutive activation of Wnt canonical pathway in human colorectal cancers [16-19].

The Wnt pathway is aberrantly activated in numerous cancers [20-22], including lung cancer [23,24]. Both Wnt-1 and Wnt-2 are up-regulated in non-small cell lung cancer (NSCLC) $[25,26]$, whereas Wnt-7a is downregulated in most lung cancer cell lines and tumor tissues [27]. Co-expression of both Wnt-7a and Fzd9 inhibits cell growth of NCSLC cell lines [27]. Moreover, DVL has been shown to be over-expressed in $75 \%$ of micro-dissected NSCLC tissues [28]. Approximately 85\% of all sporadic and hereditary colorectal tumors show loss of APC function, resulting in stabilization of $\beta$ catenin $[29,30]$. Mutations of the tumor suppressor gene $\mathrm{APC}$ or $\beta$-catenin are rare in lung cancer $[31,32]$ and the Wnt pathway may be activated upstream of $\beta$-catenin [32,33]. Furthermore, both sFRP1 and WIF1 genes are reportedly silenced in lung cancer tissues [34-36]. Taken together, these studies indicate the important roles of the Wnt pathway in lung carcinogenesis.

Knowledge regarding the regulation of specific Wnts and their corresponding receptors in lung cancer is lacking. It is not known in great detail which receptors are selectively expressed or the roles they play in the pathogenesis of lung cancer. We recently found that Wnt-2 was upregulated in NSCLC [25]. Therefore, we sought to build on this finding by investigating specific Wnt/ Frizzled interactions in human cancer cell lines and in lung cancer tissue samples. We also examined whether a dnhWnt-2 construct reduces tumor growth in cancer cell lines and in a xenograft mouse model.

\section{Methods}

\section{Cell lines and tissues}

Human lung cancer cell lines A549 and A427 were obtained from American Type Culture Collections (ATCC) (Manassas, VA) and cultured in RPMI 1640 medium. Human kidney epithelial cell line 293 and human kidney transfected epithelial cell line (293T) were obtained from ATCC and cultured in Dulbecco's modified Eagle's medium (DMEM). All cell cultures were supplemented with $10 \%$ fetal bovine serum, penicillin $(100 \mathrm{IU} / \mathrm{ml})$, and streptomycin $(100 \mu \mathrm{g} / \mathrm{ml})$ and incubated in a humid incubator with $5 \% \mathrm{CO}_{2}$ at $37^{\circ} \mathrm{C}$.

Fresh lung tumor tissues and adjacent normal lung tissues from patients who underwent surgical resection for lung cancers were collected and snap-frozen in liquid nitrogen in the operating room. Tissue samples were kept at $-170^{\circ} \mathrm{C}$ in a liquid nitrogen freezer before use. The study was approved by the Committee of Human Research at the University of California and informed consent was obtained from all patients.

\section{Semi-quantitative RT-PCR and quantitative RT-PCR}

Total RNA from mouse xenografts, fresh lung cancer and paired adjacent normal tissue was extracted with TRIzol LS (Invitrogen, Carlsbad, CA). Total RNA from the various cell lines was isolated using Qiagen's RNeasy extraction method (Valencia, CA).

For semi-quantitative analysis, reverse transcriptionPCR was performed with $1 \mu \mathrm{g}$ total RNA in a GeneAmp PCR system 9700 (Applied Biosystems, Foster City, CA) using SuperScript II One-step RT-PCR with Platinum Taq (Invitrogen, Carlsbad, CA) for 25 cycles, according to the manufacturer's instructions. Primers were obtained from Operon Biotechnologies (Alameda, CA). Primer sequences for the human Wnt-2 cDNA were 5' GGATGCCAGAGCCCTGATGAATCTT-3' (Forward) and 5'-GCCAGCCAGCATGTCCTGAGAGTA-3' (reverse). Primers for the human Frizzled-8 cDNA were 5' GGACTACAACCGCACCGACCT-3' (forward), and 5' ACCACAGGCCGATCCAGAAGAC-3' (reverse). Primer sequences for human DVL-3, c-Myc, Cyclin D1 and Survivin were obtained from Operon Biotechnologies. The housekeeping gene glyceraldehyde-3-phosphate dehydrogenase (GAPDH) (5' ATGGGGAAGGTGAAGGT CGG-3' forward; and 5'-GACGGTGCCATGGAATT TGC-3', reverse) was amplified as an internal control $[18,37]$. The ratio of band intensity of Wnt-2 and Frizzled-8 between fresh lung cancer and paired adjacent normal tissues was measured using Image $J$ software (NIH, Bethesda, MD, USA).

For quantitative RT-PCR, first-strand cDNA was synthesized from total RNA by iScript cDNA synthesis (Bio-Rad, Hercules, CA) according to the manufacturer's instructions. Taqman RT-PCR analysis was performed on cDNA in a 384-well plate using Prism 7900HT Real-Time PCR System (Applied Biosystems, Foster City, CA). Primers and hybridization probes for Wnt-2 and Frizzled-8 (inventoried, chosen from the online catalog) were purchased from Applied Biosystems (Foster City, CA). The expression of each gene was assayed in triplicate and normalized to GAPDH. 


\section{Plasmid DNA constructs}

The human Wnt-2 expression construct was kindly provided by J. Kitajewski (Columbia University). The dominant-negative Wnt-2 construct was generated by PCR amplification of the full-length human Wnt-2 cDNA using primers flanking the N-terminal domain from residues 1-278. The amplified cDNA fragment was then inserted into the pEGFP-N1vector (BD Biosciences Clontech, Palo Alto, CA) upstream of the GFP epitope to generate the dnhWnt-2 construct. The rat frizzled-1 (rFzd1), rFzd2; mouse frizzled-3 (mFzd3), mFzd4, mFzd5, mFzd7, mFrizzled-8 and mFzd9 mammalian expression constructs were kindly provided by R. Nusse (Stanford University). The mFzd10 expression construct was kindly provided by E. Morrisey (University of Pennsylvania).

\section{Selection for stable clones}

Stable cell lines were generated by transfection of the expression vectors (pGFP-N1-dnhWnt-2) and control vector (pGFP-N1) into A549 and A427 cell lines using Lipofectamine 2000 (Invitrogen, Carlsbad, CA) according to the manufacturer's instructions. Transfected cells were selected by culturing in complete medium supplemented with Geneticin at $400 \mu \mathrm{g} / \mathrm{mL}$ (Invitrogen) for approximately 1 month. The stable transfectants were isolated and expanded for further analysis.

\section{TOPflash assay}

Luciferase assays for reporters were carried out using the Dual-Luciferase Reporter Assay System (Promega, Madison, WI) as reported previously [28]. Briefly, 293, 293T, A549 and A427 cell lines were plated in 96-well plates with fresh media without antibiotics $24 \mathrm{hr}$ before transfection. Lipofectamine 2000 (Invitrogen, Carlsbad, CA) was used to mediate co-transfection of pTOPflash $(0.2 \mu \mathrm{g})$ or pFOPflash $(0.2 \mu \mathrm{g})$ vectors (kindly provided by $\mathrm{H}$. Clevers, Netherlands Institute). The cell lines were co-transfected with or without the following expression constructs: Fzd, Wnt-2, dnhWnt-2 and empty vectors pcDNA3.1 (Invitrogen) or pEGFP-N1 (each at $0.2 \mu \mathrm{g}$; $0.6 \mu \mathrm{g}$ DNA in total), as indicated. The Renilla luciferase reporter vector pRL-TK $(0.02 \mu \mathrm{g})$ (Promega, Madison, WI) was simultaneously transfected as the control for transfection efficiency. TCF-mediated transcriptional activity was determined by the ratio of pTOPflash/ pFOPflash luciferase activity, each normalized to the luciferase activities of the pRL-TK reporter. Cells were harvested $48 \mathrm{hr}$ after transfection. The experiments were done in triplicate.

\section{Western blot analysis}

Whole cell lysates of cell lines were extracted with CytoBuster Protein Extraction Reagent (Novagen, Madison,
WI). Cytosolic proteins were prepared as previously described [38]. The proteins were separated on 4-15\% gradient SDS-polyacrylamide gels and transferred to Immobilon-P membranes (Millipore, Bellerica, MA). The proteins were first bound with the following primary antibodies: $\beta$-catenin (Transduction Laboratories, Lexington, KY, USA) and $\beta$-actin (Sigma Chemical, St. Louis, MO). Antigen-antibody complexes were detected by using an ECL blotting analysis system (GE Healthcare Bio-Sciences, Piscataway, NJ). The ratio of band intensity of $\beta$-catenin to $\beta$-actin was measured using Image $J$ software $(\mathrm{NIH}$, Bethesda, MD, USA).

\section{Cell proliferation and colony formation assays}

Cell proliferation was determined using the CellTiter 96 AQueous One Solution Cell Proliferation Assay (Promega, Madison, WI). Briefly, A549 cells were plated in a 6-well plate $24 \mathrm{hr}$ before transfection. Transient transfection was carried out using $4 \mu \mathrm{g}$ of the dnhWnt-2 construct or the pEGFP-N1 empty vector. Twenty-four hours after transfection, cells were seeded in a 96-well plate at a density of $5 \times 10^{2}$ cells per well and cultured for another $24 \mathrm{hr}$ period before the CellTiter 96 Aqueous One solution was added. The assay was repeated daily for 4 consecutive days. Cell viability was measured at absorbance $490 \mathrm{~nm}$. Each experiment was done in triplicate and repeated at least three times. Colony formation was analyzed in stably transfected A549 and A427 cell lines. Cells $\left(5 \times 10^{2}\right)$ were plated in 6 -well cell-culture dishes and incubated in complete medium containing Geneticin $(400 \mu \mathrm{g} / \mathrm{mL})$ for a minimum of 14 days. The colonies were then stained with $0.1 \%$ crystal violet, and colonies were counted. Results were shown as the mean number of colonies formed with the presence of dnhWnt-2 or the empty vector control. Colony assays were performed a minimum of three times each.

\section{Tumor xenografts}

All in vivo experiments were performed in accordance with UCSF institutional guidelines (Institutional Animal Care and Use Committee approval number: AN08551601). Six week-old female nude mice, strain athymic $\mathrm{Nu} / \mathrm{Nu}$ (Taconic, Hudson, NY) received subcutaneous injections of $5 \times 10^{6}$ cells in $100 \mu \mathrm{l}$ of RPMI 1640, together with $25 \mu \mathrm{l}$ of Matrigel basement membrane matrix (Becton Dickinson, Bedford, MA). Mice were inoculated subcutaneously into the right flank with A549 stable clones expressing the dnhWnt-2 vector and into the left flank with A549 cells stably expressing the vector control. Tumors were measured twice weekly at their greatest length and width for approximately 6 weeks. Tumor volume was calculated 
according to $x^{2} y / 2$, where $x<y, \mathrm{x}=$ width and $\mathrm{y}=$ length, and was reported as the mean and standard deviation (SD) of five independent measurements ( $n=5$ mice each). After 43 days, tumors were resected and weighed. Total RNA was extracted from tumor tissues for RT-PCR analysis. Immunostaining against Ki67 was done on formalinfixed, paraffin-embedded tumor specimens resected from day 43 xenograft mice to access the level of cell proliferation. Briefly, antigen retrieval was achieved in citrate buffer, and then blocked, followed by incubation with rabbit monoclonal Ki67 antibody (Thermo Fisher Scientific Fremont, CA). Sections were then incubated with secondary goat anti-rabbit antibody (Vector Laboratories, INC. Burlingame, CA) and counterstained with Hematoxylin. Ki67 proliferation was determined by the percentage of cells with positive nuclear staining. Cell nuclei $(2,500)$ were counted on representative sections for each tumor type.

\section{Statistical analysis}

Statistical analysis was performed using GraphPad Prism 6.0 for Windows. The values shown represent mean \pm S.D. (error bars) of triplicate independent experiments. The difference between groups was determined by Student's $t$-tests and a $p$ value $\leq 0.05$ was considered statistically significant.

\section{Results}

\section{Wnt-2 activation of frizzled receptors}

Wnt-2 is overexpressed in multiple cancers [23], but the specificity of the Wnt-2 interaction with its receptor(s) remains largely unknown. We therefore investigated Wnt-2 specificity by analyzing the abilities of several Frizzled receptors to induce $\mathrm{T}$ cell factor (TCF)dependent transcription in the presence of Wnt-2. When Wnt-2 was co-expressed with each of the Frizzled receptors in 293 T cells, TCF activity of Frizzled- 8 increased by at least 25 fold over that of vector alone (Figure 1A). In addition, TCF activity of Fzd9 increased by $\sim 15$ fold over that of vector control alone, affirming previously reported data [39]. Frizzled-7 showed a 4-fold increase in TCF-activity compared to vector control and about a 2-fold increase due to the presence of Wnt-2.
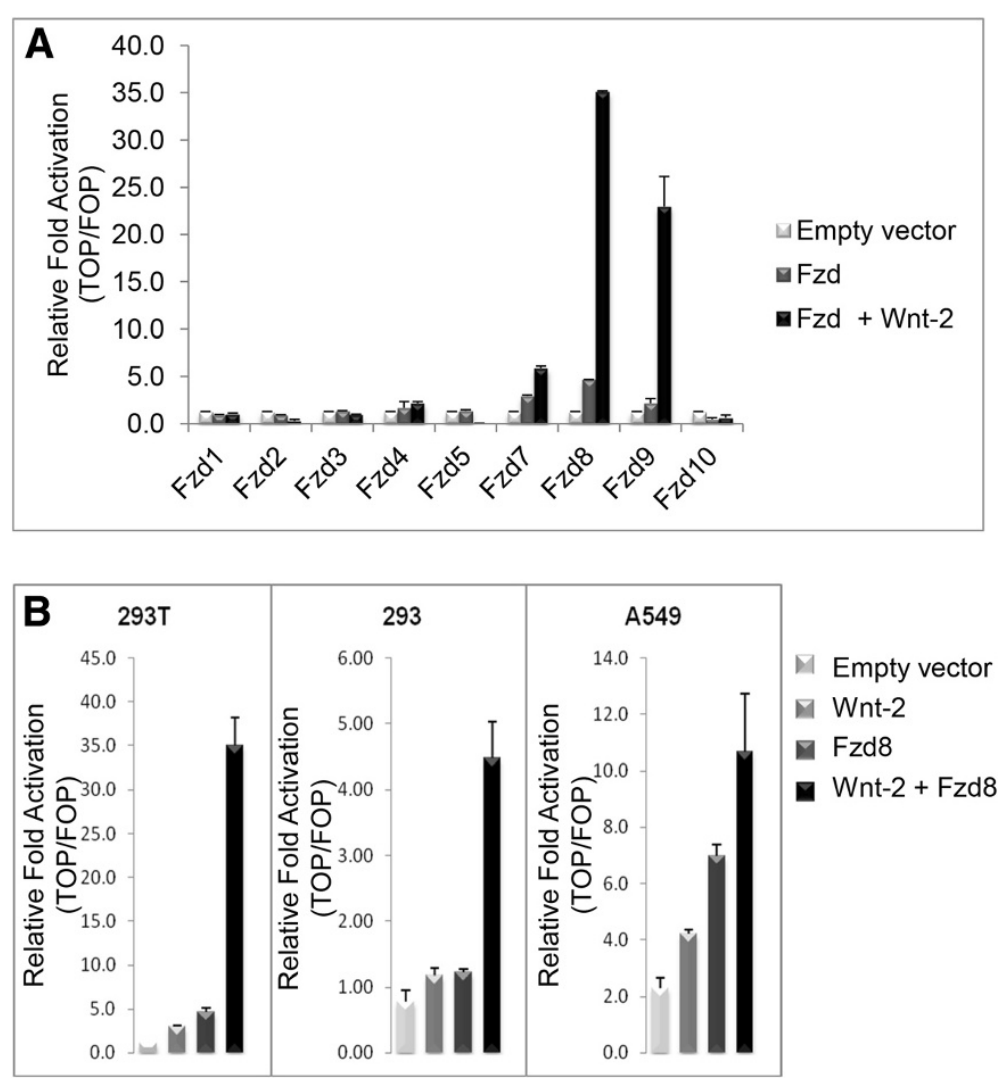

Figure 1 Wnt-2 activation of Frizzled receptors. (A) TCF transcriptional activity was measured in $293 T$ cells transfected with the indicated Frizzled expression vectors in the presence or absence of Wnt-2 cDNA and vector control. Cells were co-transfected with pTOPflash or pFOPflash and internal control plasmid pRL-TK. Experiments were performed in triplicate and the level of expression was shown as relative fold activation (TOP/FOP) (mean \pm standard deviation). (B) Activation of Frizzled-8 by Wnt-2 was measured in 293T, 293 and NSCLC cell line A549. TCF activity was determined in the cells transfected with Frizzled-8, Wnt-2, both Frizzled-8 and Wnt-2, or vector control. Experiments were performed in triplicate. 
None of the other Frizzled expression vectors (Frz 3,4,5, etc.) showed increased activation after Wnt-2 co-expression (Figure 1A). We further analyzed this activation in normal epithelial 293 cells and NSCLC cell line A549. Wnt-2 activation of Frizzled-8 increased 5-fold in these cell lines compared to that of vector control $(p<0.01)$ (Figure 1B). The empty vector control in A549 showed some activity, which is probably due to the intrinsic Wnt signaling in this cancer cell line. The results demonstrate for the first time that there is an interaction between Wnt-2 and Frizzled-8 in cancer cells.

\section{Up-regulation of Wnt-2 and frizzled-8 in lung cancer tissues}

The lung cancer tissues analyzed comprised 36 pairs of adenocarcinomas, 10 pairs of squamous cell carcinomas and 4 pairs of large cell carcinomas. Semi-quantitative RT-PCR analysis showed that Wnt-2 was up-regulated by $70 \%$ and human Frizzled- 8 was up-regulated by $42 \%$ in the 50 lung tumor samples compared to their matched normal tissue controls. Furthermore, among the 21 lung tumor samples that had Frizzled-8 upregulation, 91\% showed up-regulation of Wnt-2 $(p<0.05)$ (Figure 2).

\section{Inhibition of Wnt-2 signaling by dnhWnt-2}

We next sought to inhibit the effects of Wnt-2 activation of Frizzled- 8 by designing a novel dnhWnt- 2 construct. The human Wnt-2 gene was truncated at amino acid position 278, resulting in an 82 residue carboxyl terminal deletion generating the dnhWnt-2 construct. Coexpression of the dnhWnt-2 construct together with Wnt-2 and Frizzled-8 expression vectors in 293T and 293 cells strongly reduced TCF-dependent transcriptional activity, as determined by the TOPflash assay (Figure 3A). The 25-fold level of activation of Frizzled- 8 by Wnt-2 observed in 293T cells was reduced to near vector control levels. Similarly, activation of Frizzled- 8 by Wnt- 2 in the 293 cell line was reduced. We further analyzed this activation in NSCLC cell line A549, and observed a decrease of TCF-dependent transcriptional activity by dnhWnt-2. The dnhWnt-2 alone inhibited the intrinsic Wnt (most likely Wnt-2) signaling and resulted in the low background of TCF activity in A549 cell line (Figure 3A). To determine if the dnhWnt- 2 construct also affected $\beta$ catenin stabilization, we analyzed cytosolic $\beta$-catenin protein levels (Figure 3B). In all cell lines, $\beta$-catenin protein levels were elevated when cDNA of Frizzled- 8 and Wnt-2 were co-expressed. However, dnhWnt-2 construct reduced cytosolic $\beta$-catenin protein levels to near background levels, even when Frizzled- 8 and Wnt- 2 were coexpressed.

Effects of the dnhWnt-2 inhibitor in cancer cell lines

Since the dnhWnt-2 construct inhibited Wnt-2 signaling mediated by the Frizzled- 8 receptor, we further investigated whether the dnhWnt-2 construct could inhibit cancer cell growth. Quantitative real-time RT-PCR

\begin{tabular}{c|c|c|c|c|c|c|c|} 
Patient & 1 & 2 & 3 & 4 & 5 & 6 & 7 \\
Wnt2 & - & - & - & & - & - & - \\
FZD8 & - & - & - & - & - & - & - \\
GAPDH & $-\infty$ & -
\end{tabular}

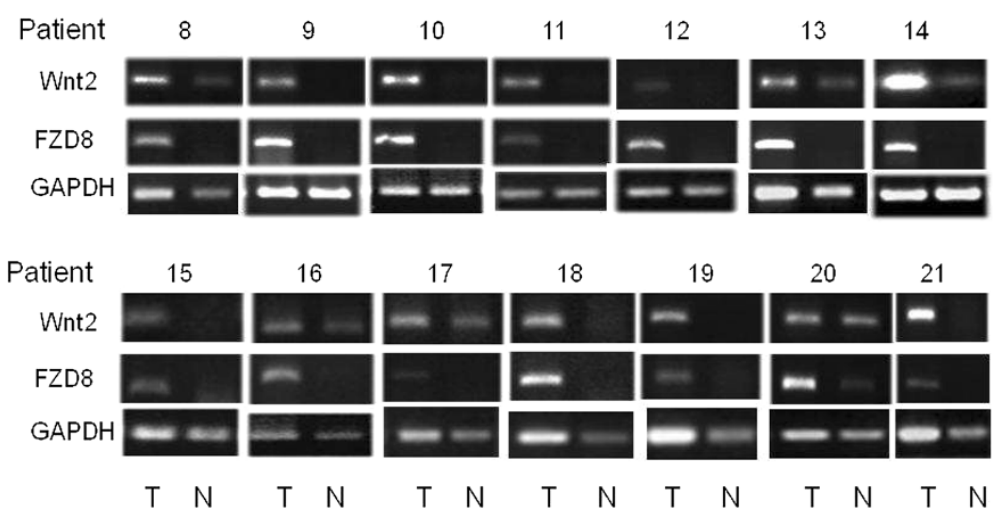

Figure 2 Up-regulation of Frizzled-8 and Wnt-2 in lung cancer. Semi-quantitative RT-PCR analysis of 50 freshly resected tumor samples with their corresponding matched, normal lung controls. Total RNA was reverse transcribed and amplified with primers specific for Wnt-2 or Frizzled-8. The data shown are representative tumor pairs (tumor $(\mathrm{T})$ and normal $(\mathrm{N})$ ). Semi-quantitative RT-PCR products were resolved on a $1.5 \%$ agarose gel. Experiments were performed in triplicate. 

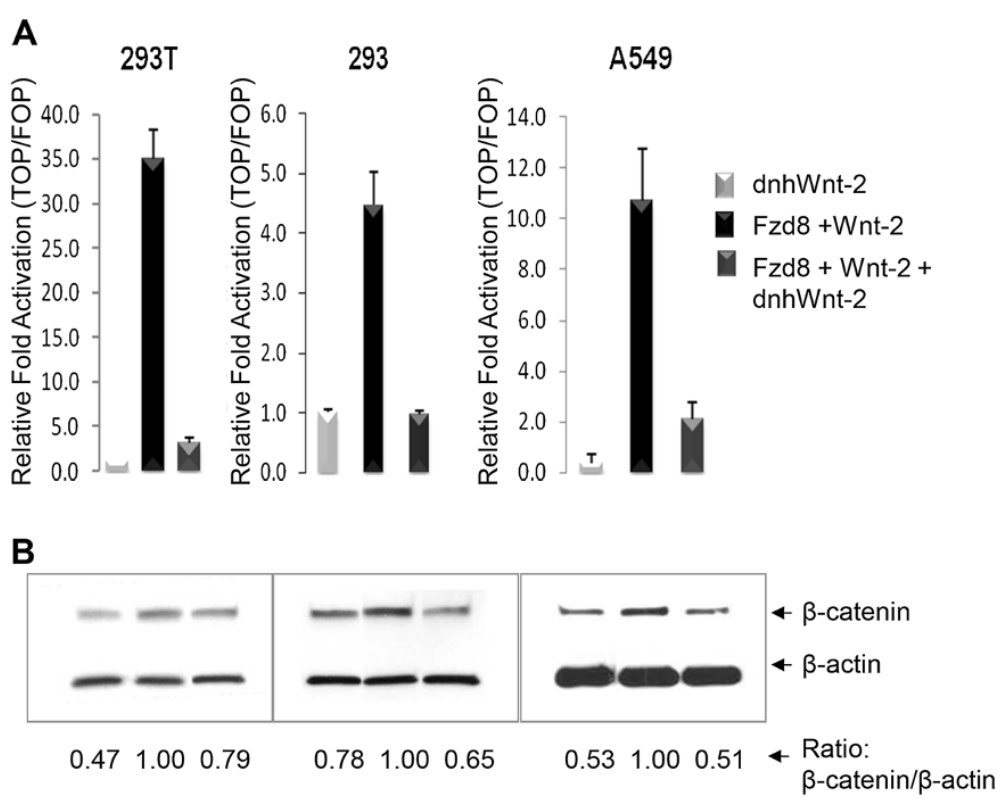

Figure 3 Dominant negative human Wnt-2 inhibits Frizzled-8 activation. (A) Inhibition of TCF transcriptional activity by dnhWnt-2 construct was measured in 293T, 293 and A549 cells. In the present or absence of dnhWnt-2 construct, the cells were co-transfected with both Frizzled-8 and Wnt-2 expression vectors, either pTOPflash or pFOPflash, and internal control plasmid pRL-TK. Experiments were performed in triplicate. (B) Stabilization of $\beta$-catenin by dnhWnt-2 expression in the indicated cell lines was determined by measuring cytosolic $\beta$-catenin protein levels in the cells.

confirmed that Wnt-2 and Frizzled-8 were endogenously overexpressed in NSCLC cell line A549 (Figure 4A) compared to normal epithelial 293 and 293 T cells. A cell proliferation assay measured over a consecutive 4-day period in A549 cells showed that dnhWnt-2 mutant inhibited cell growth (Figure 4B). Wnt-2 was expressed in NSCLC cell lines A549 and A427, which were stably transfected with the dnhWnt-2 expression vector or the vector control vector. When dnhWnt-2 was expressed, the colony formation was reduced by $52 \%$ in the A549 cell line and was not affected in the A427 cell line (Figure 5A, top). PCR primers, which are specific to the sequence presented on both Wnt-2 and the dnhWnt-2 construct, were used for semi-quantitative RT-PCR analysis, and the expression of dnhWnt-2 and the endogenous Wnt-2 in A549 and A427 cells was confirmed (Figure 5A, bottom). TCF-mediated transcription was performed on the stable cell lines (Figure 5B). A549 cells expressing the dnhWnt-2 gene showed a $36 \%$ decrease $(\mathrm{p}<0.02)$ in activity compared to vector control cells. Based on our results, we have generated two hypothetical models. Model of Wnt-2 signaling in A549 cells (Figure 5C) shows that Wnt-2 binds to the Frizzled- 8 receptor and activates Wnt-2 signaling in A549 cells (Figure 5C, left panel). The model also shows that dnhWnt-2 construct completes the binding with Wnt-2, resulting in the degradation of downstream $\beta$-catenin and the inhibition of TCF activity in A549 cells (Figure 5C, right panel). A model of Wnt-2 signaling in A427 cells (Figure 5D) shows that $\beta$-catenin mutant constitutively activates downstream Wnt signaling regardless of the presence of Wnt-2 ligand.

\section{Xenograft mouse model}

A xenograft mouse model was generated with A549 cells stably expressing the dnhWnt-2 construct and vector control plasmid. The cells were transplanted into female athymic $\mathrm{Nu} / \mathrm{Nu}$ mice and tumor formation was monitored twice per week. Tumor size and mass decreased significantly in the dnhWnt-2 tumors compared to tumor controls $(\mathrm{n}=5)$ after 43 days of growth $(p<0.05)$ (Figure 6A and 6B). Immunohistochemistry staining on tumor sections with Ki67 demonstrated cell proliferation at $\sim 80 \%$ in control tumors compared to $\sim 28 \%$ in dnhWnt-2 tumors ( $>2000$ cell counts) (Figure 6C). Further analysis of the expression of Wnt downstream target genes in the dnhWnt-2 tumors (Figure 6D) showed that the expression of Survivin, c-Myc, Dvl-3 and Cyclin-D1 genes was down-regulated in dnhWnt-2 tumors compared to control tumors.

\section{Discussion}

Wnt signaling is dysregulated in various tumors [21,22] and Wnt-2 has been suggested to play an oncogenic role in cancer [25,40]. Inhibition of Wnt signaling using different approaches has shown antitumor activity [22]. For 

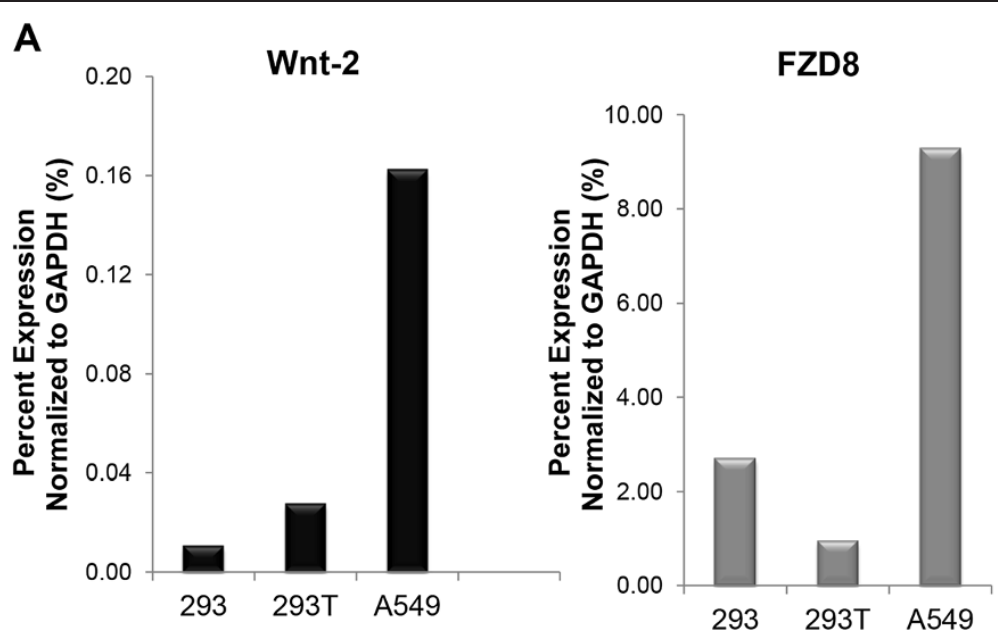

B

A549

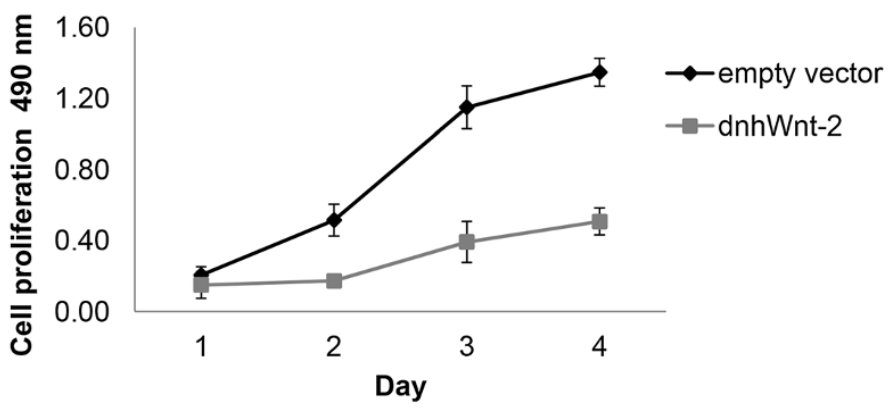

Figure 4 Endogenous expression levels of Wnt-2/Frizzled-8 and effects of dnhWnt-2 expression in normal epithelial cells and NSCLC cells. (A) Endogenous expression levels of Wnt-2 and Frizzled-8 were determined by quantitative RT-PCR analysis in 293, 293 T and A549 cells. (B) Cell proliferation assays were performed in A549 cells transfected with dnhWnt-2 or vector control plasmids.

instance, we previously reported that inhibition of Wnt2 signaling using siRNA induces programmed cell death in NSCLC cells [25]. In the current study, we demonstrated for the first time that Wnt-2 signaling is activated through the Frizzled- 8 receptor in NSCLC cells, and that a novel dnhWnt-2 construct reduces tumor growth in NSCLC cells and in a xenograft mouse model.

More recently, activation of Wnt signaling has been implicated in the metastasis of human cancer. In lung adenocarcinoma, activation of Wnt signaling has been shown to be a determinant of metastasis to brain and bone [41]. Moreover, enrichment of the Wnt-2 gene in circulating tumor cells was identified using RNA sequencing [40]. The association of Wnt-2 up-regulation with the formation of non-adherent tumors further suggests that Wnt-2 regulates metastasis of adherent tumors [40]. Our results suggest that therapeutic strategies targeting Wnt-2 signaling may prevent the development of metastasis and have potential impact on cancer mortality.

A dominant negative Wnt-8 construct has been shown to inhibit axis duplication induced by Wnt in the Xenopus model [42]. In our study, the dnhWnt-2 construct was designed by deleting an 82 amino acid truncation in the carboxyl-terminal of the human Wnt-2 gene. In our model, we demonstrated that dnhWnt-2 construct competes for the binding to the receptor(s) with Wnt-2, resulting in the degradation of cytoslolic $\beta$-catenin and the inhibition of TCF transcription in A549 cells (Figure 5C). In addition, our data indicate that the presence of dnhWnt-2 construct decreased cell proliferation and colony formation of A549 cells in vitro. We further analyzed the effect of dnhWnt-2 construct in lung cancer cell line A427, which harbors a mutation in the $\beta$-catenin gene and constitutively activates the $\beta$-catenin mutant (Figure 5D) [33]. As expected, dnhWnt-2 construct had a minimal effect on Wnt-2 signaling and colony formation in A427 cells. Although Wnt-2 is also expressed in A427 cells, its canonical signaling is probably more dependent on the $\beta$-catenin mutation and less dependent on the upstream signaling by Wnt ligands [43].

Although the frizzled family of receptors are known to function as key components of the Wnt signaling pathway [44], specific interactions of Wnt-2 with its receptor(s) 
A

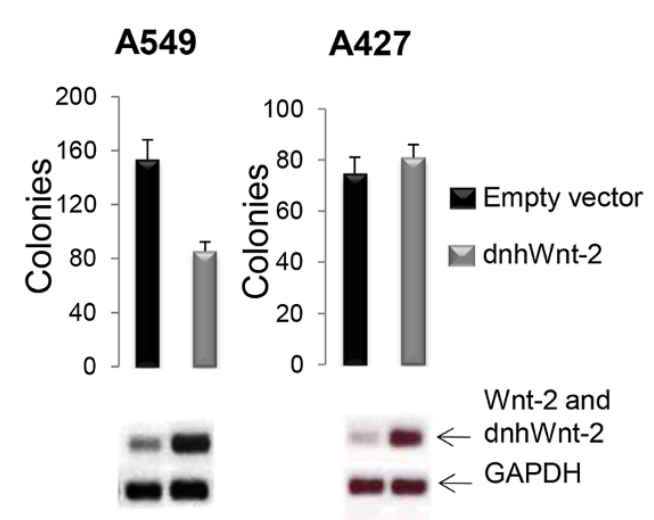

C

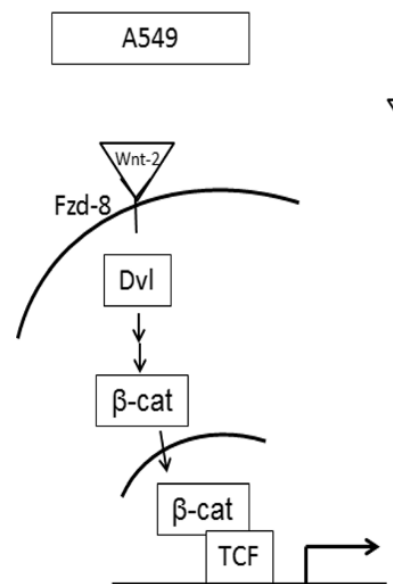

B

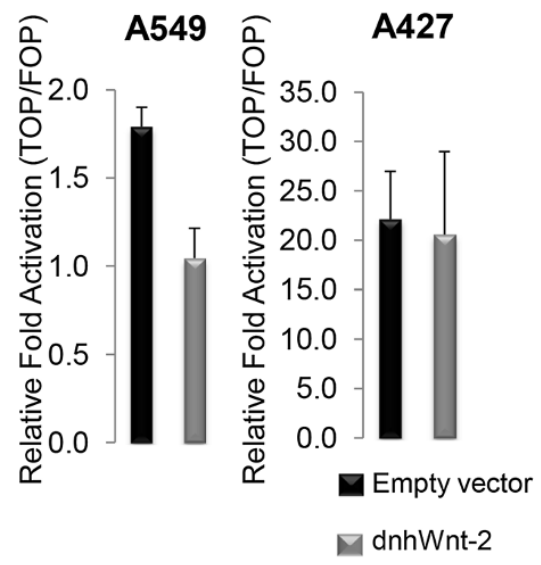

D

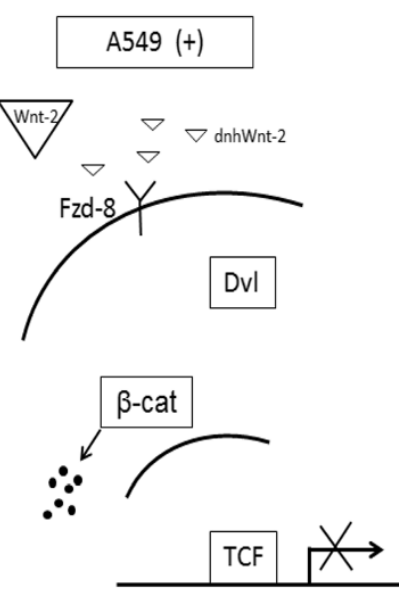

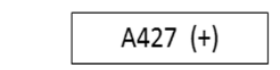
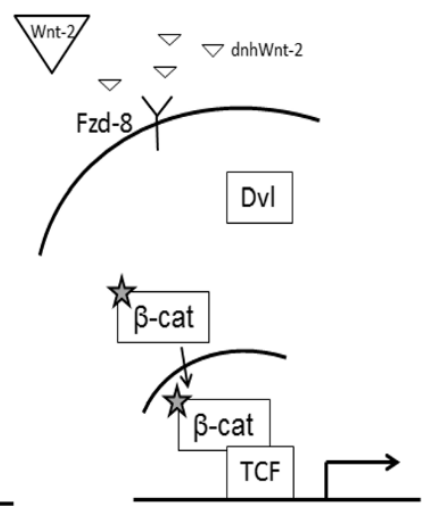

Figure 5 Effects of dnhWnt-2 expression in NSCLC cell lines. (A) Colony formation was determined in NSCLC cell lines A549 and A427. Expression of dnhWnt-2 construct and endogenous Wnt-2 was detected using semi-quantitative RT-PCR in these cell lines. (B) TCF transcriptional activity was measured in A549 and A427 cells in the present of dnhWnt-2 or vector control. Triplicate measurements were made and the level of expression was shown as relative fold activation (TOP/FOP) (mean \pm standard deviation). (C) Models of Wnt-2 signaling regulated in NSCLC cell line A549. The Wnt canonical pathway is activated when endogenous Wnt-2 ligand (large triangle) binds to Frizzled-8 receptor (Fzd-8), which recruits the intracellular protein disheveled (Dvl) to plasma membrane. Activation of Wnt canonical pathway prevents the phosphorylation of $\beta$ catenin, resulting in the stabilization and translocation of $\beta$-catenin in the nucleus, where it activates target genes through binding to TCF transcription factors. In the presence (+) of dnhWnt-2 construct (small triangle), endogenous Wnt-2 is prevented from binding to Frizzled-8 receptor. The activation of Wnt canonical pathway is inhibited, resulting in the degradation of $\beta$-catenin and blockage of TCF transcriptional activity. (D) A model demonstrates Wnt-2 signaling regulation in NSCLC cell line A427, which harbors a $\beta$-catenin mutant (star). In A427 cells, the dnhWnt-2 construct competes the binding of Frizzled-8 receptor with endogenous Wnt-2 and inhibits the activation of Wnt canonical pathway. Instead of being phosphorylated and degraded, the $\beta$-catenin mutant is constitutively expressed and activates downstream Wnt signaling regardless of the presence of Wnt-2- ligand.

have not been determined in lung cancer. In this study, we investigated the activation of Wnt-2 signaling through different Frizzled receptors. Our results show that both Frizzled-8 and Frizzled-9 were activated when Wnt-2 signaling was present in 293T cells. Overexpression of Frizzled-8 has been observed in lung cancer tissues and cell lines [45], and inhibition of Frizzled-8 expression using shRNA has been shown to reduce the proliferation of tumor cells in vitro and in a xenograft mouse model [45]. Frizzled-8 has been suggested to regulate Wnt signaling in lung cancer and can serve as a putative therapeutic target for the disease [45]. Frizzled-9 has also been shown to play a role in Wnt signaling. Rat Frizzled-9 receptor is activated by Wnt- 2 and triggers the Wnt canonical pathway in 293T cells [39], which is consistent with our observation. Frizzled-9 is also activated in Wnt-7a signaling and functions as a tumor suppressor in lung cancer [27,46]. 


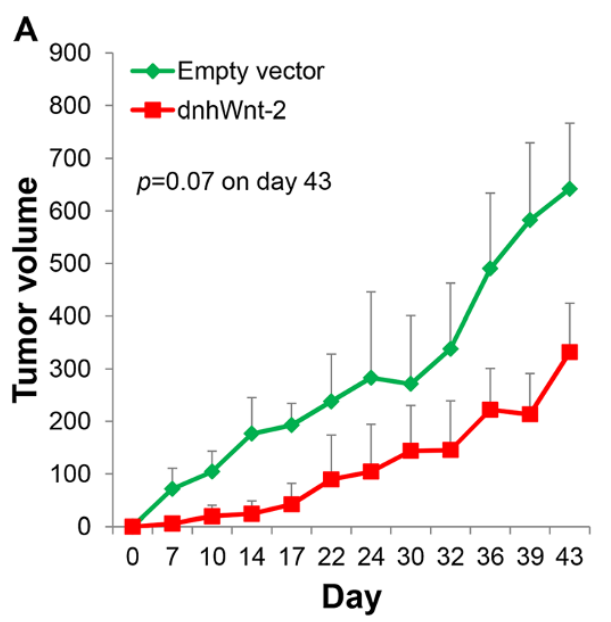

C

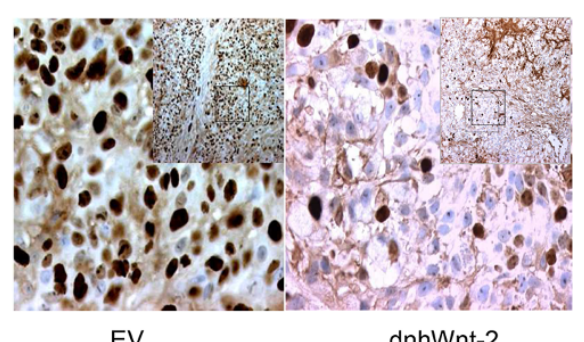

B

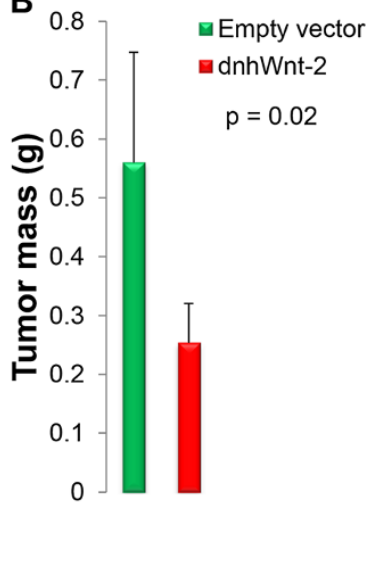

D

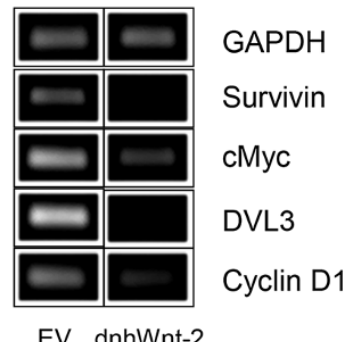

Figure 6 Tumor xenograft. (A) Tumor formation was monitored twice-weekly over a 43-day period in five groups of mice that received subcutaneous injections of A549 cells, which express dnhWnt-2 or vector control expression constructs (mean \pm standard deviation, $p=0.007$ ). (B) Tumor mass was measured on day 43 (mean \pm standard deviation, $p=0.02$ ). (C) Immunohistochemical analysis of cell proliferation by Ki-67 staining was performed in tumor sections expressing vector control (left panel) or the dnhWnt-2 (right panel) at 40x magnification. Inset is at 10x magnification. (D) qRT-PCR analysis of Wnt downstream target genes Survivin, c-Myc, DVL3, and Cyclin D1 in A549 xenograft tumors with either dnhWnt-2 or vector control. Expression of GAPDH was used as a control transcript.

Whether the activation of Frizzled-9 receptor in Wnt-2 signaling is to promote or suppress the development of lung cancer is unknown. In addition to its role in oncogenesis, Frizzled-9 mediates the activation of Wnt-7a signaling in several developmental processes in normal tissue [47-49]. The function of Frizzled-9 in Wnt signaling is complex and its role in cancer development is not clear. In addition, Wnt3a was shown to signal through multiple Frizzled receptors in 293T cells [50], and Frizzled-5 appears to be the most active receptor for Wnt3a. In human cancer, Wnt3a appears to function both as oncogene and tumor suppressor gene in different cancer cell lines [51,52]. Further studies are needed to investigate the role of Wnt3a in lung cancer.

Inhibition of Wnt signaling has been shown to reduce tumor growth in vitro and in mouse models using a variety of approaches [25,47,53-58]. For instance, small molecules have been used to inhibit Wnt secretion or the transportation of $\beta$-catenin from the nucleus $[47,55]$, and siRNA has been used to inhibit Wnt-2 signaling and induce apoptosis in NSCLC cells [25]. Fusion of Frizzled-8 CRD to human Fc can function as a soluble receptor in vivo and has been shown to inhibit tumor growth in xenograft models [59]. This antitumor activity mediated by Frizzled-8 CRD could partially result from the inhibition of Wnt-2 signaling. In this study, we used the dnhWnt-2 construct as a novel approach against lung cancer. Our results clearly show that the dnhWnt-2 construct reduces tumor growth in NSCLC cells and in a xenograft mouse model. Together, our findings, and those from other studies [25,59] strongly suggest that the further development of dnhWnt-2 construct will be useful in treating lung cancer.

\section{Conclusions}

Our study demonstrates a strong correlation between the expression of Frizzled-8 and Wnt-2 in lung tumor samples. A robust TCF-dependent transcriptional activation in cell lines was observed when both Wnt-2 and Frizzled-8 are overexpressed. A novel dnhWnt-2 construct was designed and used to inhibit TCF-mediated transcription and colony formation when expressed in NSCLC cell line A549. Moreover, the dnhWnt-2 construct reduced tumor formation and the transcription of 
downstream target genes in a xenograft mouse model. Inhibition of Wnt-2 signaling with dnhWnt-2 construct may provide a new therapeutic avenue for targeting the Wnt pathway in lung cancer.

\author{
Abbreviations \\ Fzd: Frizzled; NSCLC: Non-small-cell lung cancer; dnhWnt-2: Dominant \\ negative Wnt-2; CRD: Cysteine-rich Wnt binding domain; DVL: Dishevelled; \\ TCF: T-cell factor.
}

\section{Competing interests}

The authors have no declared conflicts of interest.

\section{Authors' contributions}

Conceived and designed the experiments: DB LY. Performed the experiments: DB KK ZX. Analyzed the data: DB KK MH ZX LY YY DJ. Wrote the paper: DB YY LY. All authors read and approved the manuscript.

\section{Acknowledgements}

This study was supported by NIH grant F32CA119636 (to D.T.B.) and R01 CA140654-01A1 (to L.Y.). We are also grateful for support from the Larry Hall and Zygielbaum Memorial Trust and Kazan, McClain, Abrams, Fernandez, Lyons, Greenwood, Harley \& Oberman Foundation, Inc., the Estate of Robert Griffiths; the Jeffrey and Karen Peterson Family Foundation; Paul and Michelle Zygielbaum; the Estate of Norman Mancini; and the Barbara Isackson Lung Cancer Research Fund. The funders had no role in study design, data collection and analysis, decision to publish, or preparation of the manuscript. Special thanks to Pamela Derish MA, Scientific Publications Manager, UCSF Department of Surgery for editing the manuscript.

Received: 7 March 2013 Accepted: 20 June 2013

Published: 1 July 2013

\section{References}

1. Maslyar DJ, Jahan TM, Jablons DM: Mechanisms of and potential treatment strategies for metastatic disease in non-small cell lung cancer. Semin Thorac Cardiovasc Surg 2004, 16(1):40-50.

2. Smith W, Khuri FR: The care of the lung cancer patient in the 21st century: a new age. Semin Oncol 2004, 31(2 Suppl 4):11-15.

3. Adesina AM, Lopez-Terrada D, Wong KK, Gunaratne P, Nguyen Y, Pulliam J, Margolin J, Finegold MJ: Gene expression profiling reveals signatures characterizing histologic subtypes of hepatoblastoma and global deregulation in cell growth and survival pathways. Hum Pathol 2009, 40(6):843-853.

4. Dann CE, Hsieh JC, Rattner A, Sharma D, Nathans J, Leahy DJ: Insights into Wnt binding and signalling from the structures of two Frizzled cysteinerich domains. Nature 2001, 412(6842):86-90.

5. Munemitsu S, Albert I, Souza B, Rubinfeld B, Polakis P: Regulation of intracellular beta-catenin levels by the adenomatous polyposis coli (APC) tumor-suppressor protein. Proc Natl Acad Sci U S A 1995, 92(7):3046-3050.

6. Liu C, Li Y, Semenov M, Han C, Baeg GH, Tan Y, Zhang Z, Lin X, He X: Control of beta-catenin phosphorylation/degradation by a dual-kinase mechanism. Cell 2002, 108(6):837-847.

7. Sakanaka C, Leong P, Xu L, Harrison SD, Williams LT: Casein kinase iepsilon in the wnt pathway: regulation of beta-catenin function. Proc Natl Acad Sci U S A 1999, 96(22):12548-12552.

8. Pinson KI, Brennan J, Monkley S, Avery BJ, Skarnes WC: An LDL-receptorrelated protein mediates Wnt signalling in mice. Nature 2000, 407(6803):535-538.

9. Tamai K, Semenov M, Kato Y, Spokony R, Liu C, Katsuyama Y, Hess F, SaintJeannet JP, He X: LDL-receptor-related proteins in Wnt signal transduction. Nature 2000, 407(6803):530-535.

10. de La Coste A, Romagnolo B, Billuart P, Renard CA, Buendia MA, Soubrane O, Fabre M, Chelly J, Beldjord C, Kahn A, et al: Somatic mutations of the beta-catenin gene are frequent in mouse and human hepatocellular carcinomas. Proc Natl Acad Sci U S A 1998, 95(15):8847-8851.

11. Behrens J, von Kries JP, Kuhl M, Bruhn L, Wedlich D, Grosschedl R, Birchmeier W: Functional interaction of beta-catenin with the transcription factor LEF-1. Nature 1996, 382(6592):638-642.
12. Huber O, Korn R, McLaughlin J, Ohsugi M, Herrmann BG, Kemler R: Nuclear localization of beta-catenin by interaction with transcription factor LEF-1. Mech Dev 1996, 59(1):3-10.

13. He TC, Sparks AB, Rago C, Hermeking H, Zawel L, da Costa LT, Morin PJ, Vogelstein B, Kinzler KW: Identification of c-MYC as a target of the APC pathway. Science 1998, 281(5382):1509-1512.

14. Tetsu O, McCormick F: Beta-catenin regulates expression of cyclin D1 in colon carcinoma cells. Nature 1999, 398(6726):422-426.

15. Wodarz A, Nusse R: Mechanisms of Wnt signaling in development. Annu Rev Cell Dev Biol 1998, 14:59-88.

16. Shimizu H, Julius MA, Giarre M, Zheng Z, Brown AM, Kitajewski J: Transformation by Wnt family proteins correlates with regulation of beta-catenin. Cell Growth Differ 1997, 8(12):1349-1358.

17. Caldwell GM, Jones C, Gensberg K, Jan S, Hardy RG, Byrd P, Chughtai S, Wallis Y, Matthews GM, Morton DG: The Wnt antagonist sFRP1 in colorectal tumorigenesis. Cancer Res 2004, 64(3):883-888.

18. Lin YC, You L, Xu Z, He B, Yang CT, Chen JK, Mikami I, Clement G, Shi $Y$, Kuchenbecker $K$, et al: Wnt inhibitory factor-1 gene transfer inhibits melanoma cell growth. Human gene therapy 2007, 18(4):379-386.

19. Sato H, Suzuki H, Toyota M, Nojima M, Maruyama R, Sasaki S, Takagi H, Sogabe $Y$, Sasaki Y, Idogawa M, et al: Frequent epigenetic inactivation of DICKKOPF family genes in human gastrointestinal tumors. Carcinogenesis 2007, 28(12):2459-2466.

20. Polakis P: The many ways of Wnt in cancer. Curr Opin Genet Dev 2007, 17(1):45-51.

21. Reya T, Clevers H: Wnt signalling in stem cells and cancer. Nature 2005, 434(7035):843-850.

22. Takebe $N$, Harris $P J$, Warren RQ, Ivy SP: Targeting cancer stem cells by inhibiting Wnt, Notch, and Hedgehog pathways. Nature Reviews Clinical Oncology 2011, 8(2):97-106.

23. Mazieres J, He B, You L, Xu Z, Jablons DM: Wnt signaling in lung cancer. Cancer Lett 2005, 222(1):1-10.

24. Van Scoyk M, Randall J, Sergew A, Williams LM, Tennis M, Winn RA: Wnt signaling pathway and lung disease. Trans/ Res 2008, 151(4):175-180

25. You L, He B, Xu Z, Uematsu K, Mazieres J, Mikami I, Reguart N, Moody TW, Kitajewski J, McCormick F, et al: Inhibition of Wnt-2-mediated signaling induces programmed cell death in non-small-cell lung cancer cells. Oncogene 2004, 23(36):6170-6174.

26. He B, You L, Uematsu K, Xu Z, Lee AY, Matsangou M, McCormick F, Jablons DM: A monoclonal antibody against Wnt-1 induces apoptosis in human cancer cells. Neoplasia 2004, 6(1):7-14.

27. Winn RA, Marek L, Han SY, Rodriguez K, Rodriguez N, Hammond M, Van Scoyk M, Acosta H, Mirus J, Barry N, et al: Restoration of Wnt-7a expression reverses non-small cell lung cancer cellular transformation through frizzled-9-mediated growth inhibition and promotion of cell differentiation. J Biol Chem 2005, 280(20):19625-19634.

28. Uematsu K, He B, You L, Xu Z, McCormick F, Jablons DM: Activation of the Wnt pathway in non small cell lung cancer: evidence of dishevelled overexpression. Oncogene 2003, 22(46):7218-7221.

29. Rubinfeld B, Souza B, Albert I, Muller O, Chamberlain SH, Masiarz FR, Munemitsu S, Polakis P: Association of the APC gene product with betacatenin. Science 1993, 262(5140):1731-1734.

30. Su LK, Vogelstein B, Kinzler KW: Association of the APC tumor suppressor protein with catenins. Science 1993, 262(5140):1734-1737.

31. Pongracz JE, Stockley RA: Wnt signalling in lung development and diseases. Respir Res 2006, 7:15.

32. Ueda M, Gemmill RM, West J, Winn R, Sugita M, Tanaka N, Ueki M, Drabkin HA: Mutations of the beta- and gamma-catenin genes are uncommon in human lung, breast, kidney, cervical and ovarian carcinomas. Br J Cancer 2001, 85(1):64-68.

33. Sunaga N, Kohno T, Kolligs FT, Fearon ER, Saito R, Yokota J: Constitutive activation of the Wnt signaling pathway by CTNNB1 (beta-catenin) mutations in a subset of human lung adenocarcinoma. Genes Chromosomes Cancer 2001, 30(3):316-321.

34. Fukui T, Kondo M, Ito G, Maeda O, Sato N, Yoshioka H, Yokoi K, Ueda Y, Shimokata K, Sekido Y: Transcriptional silencing of secreted frizzled related protein 1 (SFRP 1) by promoter hypermethylation in non-smallcell lung cancer. Oncogene 2005, 24(41):6323-6327.

35. Wissmann C, Wild PJ, Kaiser S, Roepcke S, Stoehr R, Woenckhaus M, Kristiansen G, Hsieh JC, Hofstaedter F, Hartmann A, et al: WIF1, a 
component of the Wnt pathway, is down-regulated in prostate, breast, lung, and bladder cancer. J Pathol 2003, 201(2):204-212.

36. Mazieres J, He B, You L, Xu Z, Lee AY, Mikami I, Reguart N, Rosell R, McCormick F, Jablons DM: Wnt inhibitory factor- 1 is silenced by promoter hypermethylation in human lung cancer. Cancer Res 2004, 64(14):4717-4720.

37. Kim J, You L, Xu Z, Kuchenbecker K, Raz D, He B, Jablons D: Wnt inhibitory factor inhibits lung cancer cell growth. J Thorac Cardiovasc Surg 2007, 133(3):733-737.

38. Uematsu K, Kanazawa S, You L, He B, Xu Z, Li K, Peterlin BM, McCormick F, Jablons DM: Wnt pathway activation in mesothelioma: evidence of Dishevelled overexpression and transcriptional activity of beta-catenin. Cancer Res 2003, 63(15):4547-4551.

39. Karasawa T, Yokokura H, Kitajewski J, Lombroso PJ: Frizzled-9 is activated by Wnt-2 and functions in Wnt/beta -catenin signaling. J Biol Chem 2002, 277(40):37479-37486.

40. Yu M, Ting DT, Stott SL, Wittner BS, Ozsolak F, Paul S, Ciciliano JC, Smas ME, Winokur D, Gilman AJ, et al: RNA sequencing of pancreatic circulating tumour cells implicates WNT signalling in metastasis. Nature 2012, 487(7408):510-513.

41. Nguyen DX, Chiang AC, Zhang XH, Kim JY, Kris MG, Ladanyi M, Gerald WL, Massague J: WNT/TCF signaling through LEF1 and HOXB9 mediates lung adenocarcinoma metastasis. Cell 2009, 138(1):51-62.

42. Hoppler S, Brown JD, Moon RT: Expression of a dominant-negative Wnt blocks induction of MyoD in Xenopus embryos. Genes Dev 1996 10(21):2805-2817.

43. Taketo MM: Shutting down Wnt signal-activated cancer. Nat Genet 2004, 36(4):320-322

44. van Amerongen R: Alternative Wnt pathways and receptors. Cold Spring Harb Perspect Biol 2012, 4:a007914.

45. Wang $\mathrm{HQ}$, Xu ML, Ma J, Zhang Y, Xie CH: Frizzled-8 as a putative therapeutic target in human lung cancer. Biochem Biophys Res Commun 2012, 417(1):62-66.

46. Winn RA, Van Scoyk M, Hammond M, Rodriguez K, Crossno JT Jr, Heasley $L E$, Nemenoff RA: Antitumorigenic effect of Wnt 7a and Fzd 9 in nonsmall cell lung cancer cells is mediated through ERK-5-dependent activation of peroxisome proliferator-activated receptor gamma. J Biol Chem 2006, 281(37):26943-26950.

47. David R: Developement: Strong bones: got FZD9? Nat Rev Mol Cell Biol 2011, 12(5):280.

48. Ranheim EA, Kwan HC, Reya T, Wang YK, Weissman IL, Francke U: Frizzled 9 knock-out mice have abnormal B-cell development. Blood 2005, 105(6):2487-2494.

49. Zhao C, Aviles C, Abel RA, Almli CR, McQuillen P, Pleasure SJ: Hippocampal and visuospatial learning defects in mice with a deletion of frizzled 9 , a gene in the Williams syndrome deletion interval. Development 2005, 132(12):2917-2927.

50. Liu G, Bafico A, Aaronson SA: The mechanism of endogenous receptor activation functionally distinguishes prototype canonical and noncanonical Wnts. Mol Cell Biol 2005, 25(9):3475-3482.

51. Green JL, La J, Yum KW, Desai P, Rodewald LW, Zhang X, Leblanc M, Nusse R, Lewis MT, Wahl GM: Paracrine Wnt signaling both promotes and inhibits human breast tumor growth. Proc Natl Acad Sci U S A 2013, 110(17):6991-6996

52. Chien AJ, Moore EC, Lonsdorf AS, Kulikauskas RM, Rothberg BG, Berger AJ, Major MB, Hwang ST, Rimm DL, Moon RT: Activated Wnt/beta-catenin signaling in melanoma is associated with decreased proliferation in patient tumors and a murine melanoma model. Proc Natl Acad Sci U S A 2009, 106(4):1193-1198.

53. Fujii N, You L, Xu Z, Uematsu K, Shan J, He B, Mikami I, Edmondson LR, Neale $G$, Zheng J, et al: An antagonist of dishevelled protein-protein interaction suppresses beta-catenin-dependent tumor cell growth. Cancer Res 2007, 67(2):573-579.

54. Shan J, Shi DL, Wang J, Zheng J: Identification of a specific inhibitor of the dishevelled PDZ domain. Biochemistry 2005, 44(47):15495-15503.

55. Yoshizumi T, Ohta T, Ninomiya I, Terada I, Fushida S, Fujimura T, Nishimura G, Shimizu K, Yi S, Miwa K: Thiazolidinedione, a peroxisome proliferatoractivated receptor-gamma ligand, inhibits growth and metastasis of HT29 human colon cancer cells through differentiation-promoting effects. Int J Oncol 2004, 25(3):631-639.
56. Kim MS, Kim SS, Ahn CH, Yoo NJ, Lee SH: Frameshift mutations of Wnt pathway genes AXIN2 and TCF7L2 in gastric carcinomas with high microsatellite instability. Hum Pathol 2009, 40(1):58-64.

57. Martin V, Valencia A, Agirre X, Cervera J, San Jose-Eneriz E, Vilas-Zornoza A, Rodriguez-Otero P, Sanz MA, Herrera C, Torres A, et al: Epigenetic regulation of the non-canonical Wnt pathway in acute myeloid leukemia. Cancer science 2010, 101(2):425-432.

58. Long F: Building strong bones: molecular regulation of the osteoblast lineage. Nat Rev Mol Cell Biol 2012, 13(1):27-38.

59. DeAlmeida VI, Miao L, Ernst JA, Koeppen H, Polakis P, Rubinfeld B: The soluble wnt receptor Frizzled8CRD-hFc inhibits the growth of teratocarcinomas in vivo. Cancer Res 2007, 67(11):5371-5379.

doi:10.1186/1471-2407-13-316

Cite this article as: Bravo et al.: Frizzled-8 receptor is activated by the Wnt-2 ligand in non-small cell lung cancer. BMC Cancer 2013 13:316.

\section{Submit your next manuscript to BioMed Central and take full advantage of:}

- Convenient online submission

- Thorough peer review

- No space constraints or color figure charges

- Immediate publication on acceptance

- Inclusion in PubMed, CAS, Scopus and Google Scholar

- Research which is freely available for redistribution 